\title{
Addressing the multisectoral impact of pressure injuries in the USA, UK and abroad
}

\author{
William V Padula, ${ }^{1,2}$ Peter J Pronovost ${ }^{2,3}$
}

${ }^{1}$ Department of Health Policy and Management, Bloomberg School of Public Health, The Johns Hopkins University, Baltimore, Maryland, USA ${ }^{2}$ The Armstrong Institute for Patient Safety and Quality, Johns Hopkins Medicine, Baltimore, Maryland, USA

${ }^{3}$ Department of Anesthesiology and Critical Care and

Department of Surgery, School of Medicine, The Johns Hopkins University, Baltimore, Maryland, USA

\section{Correspondence to}

Dr William V Padula, Johns Hopkins Bloomberg School of Public Health, 615 N Wolfe St, Baltimore, MD 21205, USA wpadula@jhu.edu

Accepted 6 September 2017 Published Online First

19 September 2017

\section{SLinked}

http://dx.doi.org/10.1136/ bmjqs-2017-006726

Check for updates

To cite: Padula WV, Pronovost PJ. BMJ Qual Saf 2018:27:171-173.
Pressure injuries are problematic to health systems throughout the world, significantly harming over 7 million patients and adding extraordinary costs. The USA, for instance, experiences more than 2.5 million pressure injury cases per year which cause over 60000 deathsthat is more than car accident fatalities in the USA-and cost the health system at least \$9-\$11 billion. ${ }^{1}$ The UK is no less affected by 700000 cases per year that result in 27000 deaths and cost the National Health Service (NHS) an estimated $£ 1.4-£ 2.1$ billion. $^{2}$

Despite there being no reason to expect major differences in the presentation of pressure injuries between the two countries, the UK appears mainly to have a problem with chronic pressure ulcers, whereas the USA appears to have an increasing burden of acute pressure ulcers. $^{3}{ }^{4}$ The UK NHS reports chronic wounds accounting for $78 \%$ of the NHS spending on wound treatments. ${ }^{5}$ On the other hand, the acute issue in the USA may be connected to the Centers for Medicare and Medicaid Services (CMS) reimbursement policy, which has cut payments since 2008 for hospitalised patients who acquired pressure injuries as well as penalised facilities with poorest hospital-acquired condition rates. ${ }^{67}$ This CMS policy also focuses financial responsibility of the pressure injury on the facility that identifies the wound, which means that facilities trying to correct other's mistakes are often blamed.

Like most quality measures derived from billing data that have a financial penalty, variability in pressure injury rates is a combination of changes in reporting and how clinicians care for patients by implementing a pressure injury prevention protocol. While we do not have empirical evidence for the relative proportions that these two domains contribute to pressure injury rates, our experience suggests that health systems' initial response to CMS policy has been improvements in coding and tracking pressure injuries. The more difficult, long-term improvements have been to implement better prevention protocols to actually reduce patient harm.

The study by Squitieri and colleagues in this issue of BMJ Quality \& Safety is eye opening about the appropriateness of this policy and who is responsible for the rates of pressure injuries, especially in the USA. ${ }^{8}$ Their study used Medicare claims data to evaluate agreement in pressure injury diagnosis across facilities when a patient was transferred between a community dwelling and acute care. They observed only 36.3\% agreement in the documentation of pressure injuries during a transfer between facilities, and similarly poor rates of agreement in staging. These poor levels of agreement confirm a discordance of pressure injury documentation between facilities that attempts to undermine CMS penalties on the facility that initially caused the wound. It also places question on the quality of care witnessed during the transfer process, since patients can pass between modes of transportation, emergency departments and many other healthcare settings before a clinician performs the next skin check.

One of the concerning unintended consequences of the CMS policy is inconsistent reporting. Meddings and colleagues published that reporting consistency between patient records and billing claims in hospitals was pooronly about $7.5 \%$ of pressure injuries are consistently billed that appear in patient records. $^{9}$ This discrepancy emphasises the extent of the reluctance for hospitals to voluntarily report something that can 
harm them financially and hurt their reputation in publicly reported performance statistics.

Prior to Squitieri and colleagues' study, it was not clear whether other types of facilities were facing the same issue in reporting pressure injuries, or whether hospitals were really the main culprit of pressure injury incidence in the USA. ${ }^{8}$ This new study confirms that pressure injuries may really be, in part, as much a chronic issue in the USA as in the UK, but CMS payment incentives continue to skew the index location of the wound. Long-term care and home health contingencies benefit from hospitals' inability to process patients coming from different care pathways, check for pressure injuries and properly document when the injury is present on admission, eventually making it hospitals' problems.

The poor agreement in pressure injury documentation between facilities found by Squitieri and colleagues also suggests that pressure injuries are occurring during the transfer process, or during admission through the emergency department where few patients receive skin checks or prevention protocols. ${ }^{8}$ Clinical studies indicate that for patients with highly acute malnutrition, a pressure injury can develop in a matter of hours after the patient enters the healthcare system. ${ }^{10}$ Ambulance transfers have little protocol, if any, to address pressure injury prevention when a patient is lying on a board for several hours while first responders' main priority is to maintain a patient's stability after a traumatic event. Similarly, emergency department's main priority is addressing acute problems. Patients admitted through the emergency department may spend several hours or an overnight in observation being served for the acute problem with little focus on the prevention of pressure injuries. Given the often long emergency department boarding times, a patient may not begin receiving a pressure injury prevention protocol for many hours, until well after admission to an inpatient unit, and by then it may be too late to stymie the advancement of a new pressure injury. In addition, by the time the injury is identified in the hospital, it is difficult to determine where it originated; culpability would be assigned to the hospital.

It is apparent that hospitals are in a difficult situation when it comes to being the scapegoat for most pressure injuries in the USA, and few have the bandwidth currently to upgrade their routine of skin checks and initiating a pressure injury prevention protocol in every part of the system, be it in home health, long-term care, interfacility transportation, the emergency department as well as inpatient units. The CMS payment policies have centralised the responsibility on hospitals to prevent pressure injuries or deal with multifold consequences, but this penalising system also suppresses hospitals from creating additional bandwidth to expand a prevention and skin care programme that identifies pressure injuries at the source of the problem.
Another recent study highlighted the value of cultural indoctrination of transparency as one of the first steps in bringing healthcare systems up to speed on important quality improvement efforts such as hospital-acquired condition prevention. ${ }^{11} \mathrm{~A}$ health system that knows where pressure injuries are coming from is more prepared to create a targeted infrastructure for improvement. A second step in the improvement process is leadership support to invest in stronger infrastructure where the problem lies. ${ }^{12}$ For many hospitals, if pressure injuries are actually occurring in those facilities, then hospitals need more skilled specialists (eg, board-certified wound care nurses and therapists) who can address individualised care needs for prevention and treatment of pressure injuries, and teach other bedside clinicians how to more effectively prevent pressure injuries, or at least identify early-stage, high-risk cases before they become problematic. ${ }^{13}$ We see that there is inherent value in investing short term in this specialist infrastructure, and in the long term it may save on the cost of paying for additional bedside care. Health systems also need more robust systems to accurately measure the incidence rates of pressure injuries across the continuum of care, transparently report their rates to clinicians and managers, and create shared accountability systems for improvement.

The third step is investing creatively in new technologies that can make the process of pressure injury prevention simpler through the pathways of patient care. A first responder already has many actions to take when a patient is being ambulanced into a facility, and time is critical. Yet, it might not be too difficult to place a prophylactic dressing on a patient's backside or occiput to reduce the impact of pressure and friction to high-pressure points during the transportation process. ${ }^{14}$ Ambulances and emergency department surfaces also typically have little technology in the way of pressure relief-it is like the dark ages considering pressure sensing and relieving options are widely available (though sometimes costly). ${ }^{15}$ Systems that determine the root cause of pressure injuries happen elsewhere than in the hospital should be thinking about investment in these effective technologies for emergency departments, transportation, and so on, rather than punting a high-risk case to inpatient care.

Finally, the issue presented in Squitieri and colleagues' research in combination with other recent findings illustrates that CMS payment policies have not sustained long-term reductions in pressure injuries. ${ }^{4} 8$ There is some association between these policies and pressure injury reductions, but competing evidence also suggests that systems may simply be relocating the problem or under reporting in billing claims. ${ }^{16}$

CMS needs to consider alternative payment structures that incentivise prevention and invest in a quality improvement infrastructure, such as a two-sided risk model that still penalises hospitals for poor performance, but also rewards hospitals for significant rate 
reductions. Investing in hospitals by paying for prevention would create bandwidth for hospitals to investigate quality improvement strategies that work to indoctrinate culture with transparency and a stronger preventive knowledge base. CMS could learn much in this respect from the NHS, which through The Kings Fund has allocated more than $£ 22$ billion through 2021 to invest in a sustainable quality improvement infrastructure. ${ }^{17}$ Financial incentives without sufficient skill, resources and infrastructure will predictably result in gaming rather than improving.

Contributors WVP and PJP were involved in all aspects of writing this editorial.

Competing interests None declared.

Provenance and peer review Commissioned; internally peer reviewed.

(C) Article author(s) (or their employer(s) unless otherwise stated in the text of the article) 2018. All rights reserved. No commercial use is permitted unless otherwise expressly granted.

\section{REFERENCES}

1 Lyder CH, Ayello EA. Pressure Ulcers: A Patient Safety Issue. Hughes RG, ed. Patient safety and quality: an evidence-based handbook for nurses. Rockville, MD: Agency for Healthcare Research and Quality, 2008.

2 Bennett G, Dealey C, Posnett J. The cost of pressure ulcers in the UK. Age Ageing 2004;33:230-5.

3 Jackson D, Durrant L, Bishop E, et al. Health service provision and the use of pressure-redistributing devices: mixed methods study of community dwelling individuals with pressure injuries. Contemp Nurse 2017:1-12.

4 Waters TM, Daniels MJ, Bazzoli GJ, et al. Effect of Medicare's nonpayment for Hospital-Acquired Conditions: lessons for future policy. JAMA Intern Med 2015;175:347-54.

5 Guest JF, Ayoub N, McIlwraith T, et al. Health economic burden that different wound types impose on the UK's National Health Service. Int Wound J 2017;14:322-30.
6 Wald HL, Kramer AM. Nonpayment for harms resulting from medical care: catheter-associated urinary tract infections. JAMA 2007;298:2782-4.

7 CMS Media Relations. CMS to Improve Quality of Care during Hospital Inpatient Stays. In: Department of Health o Human Services. Washington, DC: Centers for Medicare and Medicaid Services, 2014.

8 Squitieri L, Ganz DA, Mangione CM, et al. Consistency of pressure injury documentation across interfacility transfers. BMJ Qual Saf 2018;27:182-9.

9 Meddings JA, Reichert H, Hofer T, et al. Hospital report cards for hospital-acquired pressure ulcers: how good are the grades? Ann Intern Med 2013;159:505-13.

10 Gefen A. How much time does it take to get a pressure ulcer? Integrated evidence from human, animal, and in vitro studies. Ostomy Wound Manage 2008;54:2630-5.

11 Pronovost PJ, Weaver SJ, Berenholtz SM, et al. Reducing preventable harm: observations on minimizing bloodstream infections. J Health Organ Manag 2017;31:2-9.

12 Padula WV, Gibbons RD, Valuck RJ, et al. Are evidence-based practices associated with effective prevention of hospitalacquired pressure ulcers in US academic medical centers? Med Care 2016;54:512-8.

13 Padula WV, Mishra MK, Makic MB, et al. A framework of quality improvement interventions to implement evidencebased practices for pressure ulcer prevention. Adv Skin Wound Care 2014;27:280-4.

14 Padula WV. Effectiveness and value of prophylactic 5-layer foam sacral dressings to prevent hospital-acquired pressure injuries in acute care hospitals: an observational cohort study. $J$ Wound Ostomy Continence Nurs 2017;44:413-9.

15 Walia GS, Wong AL, Lo AY, et al. Efficacy of monitoring devices in support of prevention of pressure injuries: systematic review and meta-analysis. Adv Skin Wound Care 2016;29:567-74.

16 Meddings J. Using administrative discharge diagnoses to track hospital-acquired pressure ulcer incidence--limitations, links, and leaps. Jt Comm J Qual Patient Saf 2015;41:243-5.

17 Alderwick H, Ham C. Sustainability and transformation plans for the NHS in England: what do they say and what happens next? BMJ 2017;356:j1541. 